\title{
EFECTO DE LAS HELADAS TARDÍAS EN LA PRODUCCIÓN DE DURAZNEROS PRECOCES CULTIVADOS EN LA REGIÓN CENTRAL DE LA PROVINCIA DE SANTA FE (ARGENTINA)
}

\author{
García, M. S. ${ }^{1} ;$ Micheloud, N. G. ${ }^{2}$; LeVA, P. ${ }^{1 ;}$ TÓffoli, G. ${ }^{1}$; \\ Gariglio, n. F. ${ }^{3}$ \& Pilatti, R. A. ${ }^{2}$
}

\begin{abstract}
RESUMEN
El objetivo del presente trabajo fue evaluar el efecto de las heladas tardías sobre la producción de durazneros implantados en la región centro de la provincia de Santa Fe. Los cultivares estudiados fueron 'Flordastar', 'Tropic Snow', 'Early Grande', 'San Pedro', 'Hermosillo' y 'Flordaking'. Las temperaturas mínimas críticas utilizadas fueron las establecidas por Proebsting \& Mills (1978). Se registraron las heladas meteorológicas desde mayo a septiembre de los años 2007 y 2008 . El cuajado de frutos constituyó el periodo de mayor sensibilidad. Este período ocurrió en la segunda quincena de agosto. En los años 2007 y 2008 se registraron 13 y 10 heladas meteorológicas, respectivamente. En el 2007, las mermas de rendimiento fluctuaron entre el 7 y 55\%, siendo 'Tropic Snow', 'Hermosillo' y 'Early Grande' los cultivares más afectados. En el 2008, las pérdidas superaron el $80 \%$ en todos los cultivares, justificando las medidas activas de lucha contra las heladas tardías.
\end{abstract}

Palabras claves: Prunus persica, daño, temperaturas críticas, control activo de heladas.

\section{SUMMARY}

\section{Effect of late frost on fruit yield of peaches cultivated in the central area of the province of Santa Fe (Argentina).}

The aim of this study was to evaluate the effect of late frosts on the production of peaches implanted in the central region of the province of Santa Fe. The cultivars studied were 'Flordastar', 'Tropic Snow', 'Early Grande', 'San Pedro', 'Hermosillo' and 'Flordaking'. The minimum critical temperatures used were established by Proebsting \& Mills (1978). Weather frosts were recorded from May to September 2007 and 2008. The fruit set was the period of greatest sensitivity. This period occurred in the second half of August. In 2007 and 200813 and 10 weather frosts were

1.- Cátedra de Agrometeorología. Facultad de Ciencias Agrarias, Universidad Nacional del Litoral. Kreder 2805. (3080) Esperanza (Santa Fe). Tel.: (03496) 420639, int. 252. e-mail: msgarcia@fca.unl.edu.ar 2.- Cátedra de Fisiología Vegetal. FCA (UNL).

3.- Cátedra de Cultivos Intensivos. FCA (UNL).

Manuscrito recibido el 16 de diciembre de 2013 y aceptado para su publicación el 24 de febrero de 2014. 
recorded, respectively. In 2007, the yield losses ranged between 7 and 55\%. The most affected were 'Tropic Snow', 'Hermosillo' and 'Early Grande'. In 2008, losses exceeded 80\% in all cultivars, justifying active measures against late frosts.

Key words: Prunus persica, injury, critical temperatures, active frost control.

\section{INTRODUCCIÓN}

Durante varios años, en la región centro de la provincia de Santa Fe se ha evaluado el comportamiento vegetativo y reproductivo de diferentes cultivares de durazneros de bajos requerimientos de frío (Gariglio et al., 1999, 2001, 2003, 2006; Ortiz de Zárate \& Gariglio, 2004; Gariglio et al., 2009). La precocidad en el despertar vegetativo manifestada por estos cultivares de menor crioexigencia, no es acompañada por un aumento en la resistencia a heladas, por lo que el riesgo de experimentar daño por temperaturas bajo cero que afecten la producción anual tiende a incrementarse (Pascale et al., 2001).

Estudios realizados por Baldini (1992), informan que el daño producido por temperaturas congelantes en los frutales no sólo depende de las temperaturas mínimas críticas de los cultivos, sino del tipo de yema y estado fenológico en el que se encuentren. Según el autor, el ovario, los óvulos y la base del estilo en las yemas reproductivas son las partes más sensibles al frío, mientras que las cubiertas florales son las más resistentes.

Con respecto a los umbrales térmicos críticos de los frutales, es amplia la bibliografía existente (Tassara \& Charles, 1984). Estas temperaturas varían según el lugar, las condiciones meteorológicas acompañantes, la especie y el cultivar considerado, pero lo que es común para todas las situaciones, es que la intensidad de las temperaturas críticas disminuye a medida que el proceso de floración avanza, siendo el cuajado de los frutos, el estadio de máxima sensibilidad al frío (Proebsting \& Mills, 1978).

Teniendo en cuenta lo mencionado anteriormente, conocer el régimen agroclimático de heladas, las temperaturas críticas de los cultivos y las fechas fenológicas de los principales cultivares implantados en la región, son puntos importantes a tener en cuenta al explicar la merma de rendimiento producida por temperaturas congelantes y de esta manera poder justificar la implementación de lucha contra las heladas.

En este contexto se comenzó a trabajar sobre uno de los aspectos térmicos que condicionan la producción de frutas en Santa Fe, como es el riesgo de heladas primaverales (García et al., 2008). En la provincia, las heladas ocurren entre los meses de mayo y septiembre, con una frecuencia e intensidad que aumenta en el mes de julio. Las temperaturas mínimas absolutas medias, están comprendidas entre $-2,7^{\circ} \mathrm{C} \mathrm{y}-3,9^{\circ} \mathrm{C}$ (García et al., 2008), mientras que la floración y brotación a campo de los cultivares de durazno implantados se registran entre fines de julio y principios de agosto (Gariglio et al., 2012).

Por lo expuesto anteriormente es muy probable que en determinados años, en los cultivares presentes en la región, se produzcan mermas en sus rendimientos. Esto no solo dependerá de la época de ocurrencia de las heladas, sino del estado 